Vaccination before transfer to docetaxel therapy at time of disease progression increased the median progression-free survival compared with the response of the historical controls (6.1 months vs 3.7 months).

The authors conclude that docetaxel can be used safely in combination with immunotherapy, without affecting the magnitude of the tumor-specific T-cell response, and that previously vaccinated patients might have prolonged responses to docetaxel compared with unvaccinated patients.

Kate Matthews

Original article Arlen PM et al. (2006) A randomized phase II study of concurrent docetaxel plus vaccine versus vaccine alone in metastatic androgen-independent prostate cancer. Clin Cancer Res 12: 1260-1269

\section{Induction chemotherapy for laryngeal cancer selects patients for organ-preserving treatment}

A paper in the Journal of Clinical Oncology reports promising survival rates in patients with laryngeal cancer, and demonstrates that induction chemotherapy could select patients for organ-preserving treatment. In this phase II trial, patients were selected for nonsurgical treatment based on their response to a single cycle of induction chemotherapy.

A total of 97 patients with stage III or IV squamous-cell carcinoma of the larynx underwent induction therapy with fluorouracil and either cisplatin or carboplatin. A response of $>50 \%$ was achieved by 73 patients who received concurrent chemoradiation. Patients who exhibited a $<50 \%$ response to the induction regimen underwent a total laryngectomy and postoperative radiotherapy. Eight weeks after treatment, 68 patients in the chemoradiation group had no residual disease at the primary site and received two cycles of adjuvant chemotherapy, with a regimen identical to that used for induction. Patients with residual disease underwent a total laryngectomy and postoperative radiotherapy. Total overall survival and disease-free survival rates at 3 years were $85 \%$ and $78 \%$, respectively. Larynx preservation was achieved in $70 \%$ of patients. At 3 years, overall survival in this group was $61 \%$, which was comparable to that in patients who underwent laryngectomy.

The authors conclude that one cycle of neoadjuvant chemotherapy can effectively identify patients who are likely to respond to chemoradiation, and that a phase III trial to compare this treatment paradigm with standard chemoradiation regimens is warranted.

Alexandra King

Original article Urba S et al. (2006) Single-cycle induction

chemotherapy selects patients with advanced laryngeal cancer for combined chemoradiation: a new treatment paradigm. J Clin Oncol 24: 593-598

\section{Overexpression of p95HER2 in breast cancer is associated with poor prognosis}

HER2 overexpression is found in $25-30 \%$ of breast cancers and has been associated with increased risk of relapse and mortality. Studies have indicated that metastatic nodal tissue contains higher levels of p95HER2, a truncated fragment of HER2, than does primary nodal tissue. Sáez et al. have investigated whether p95HER2 can be used as a prognostic indicator in breast cancer.

Western blot analysis was used to identify HER2/neu proteins in primary breast tumor tissue samples from surgical resections in hospitals in the US $(n=334)$ and Spain $(n=149)$. Levels of p95HER2 and p185HER2 (the fulllength HER2 receptor) were scored as either high or low relative to standard values.

Overexpression of p95HER2 was found in $44 / 483$ patients $(9 \%)$, and p185HER2 overexpression in $145 / 483$ patients (30\%). Overexpression of p95HER2 was associated with various breast cancer risk factors, including negative estrogen receptor status and negative progesterone receptor status $(P=0.013$ and $P=0.001$, respectively), but p185HER2 expression levels did not correlate with these risk factors. Patients with high levels of p95HER2 were at significantly greater risk of recurrence or breast-cancer-related death than were patients with low p95HER2 levels (hazard ratio $2.55 ; P<0.0001$ ). By contrast, high levels of p185HER2 did not increase the risk of recurrence or breast-cancer-related death compared with low levels (hazard ratio 1.23; $P=0.261$ ).

The authors conclude that overexpression of p95HER2 in breast cancer might be useful for identifying patients with a poor prognosis who could benefit from adjuvant therapy.

Rebecca Ireland

Original article Sáez R et al. (2006) p95HER-2 predicts worse outcome in patients with HER-2-positive breast cancer. Clin Cancer Res 12: 424-431 